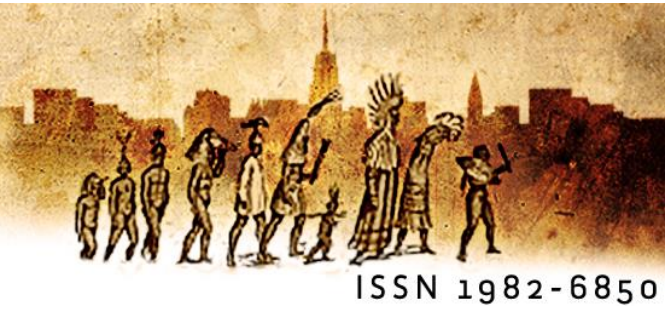

\title{
Lecture subjective : implication émotionnelle et cognitive du sujet lecteur ${ }^{1}$
}

Annie Rouxeli - (Université de Bordeaux)

\begin{abstract}
Résumé : La lecture subjective désigne une forme de lecture littéraire qui met à distance les approches encore trop exclusivement formalistes de la littérature. Elle requiert la participation du sujet lecteur et rend compte de la manière singulière dont il s'approprie le texte et le remodèle en y investissant sa personnalité profonde, ses valeurs et son imaginaire. La réflexion portera sur l'importance heuristique, la richesse, la complexité du vécu subjectif dans l'expérience de lecture. Comment s'exprime la subjectivité du sujet? Quels liens se tissent entre émotion et cognition? Enfin, comment les réactions sensibles des lecteurs conduisent-elles à des interprétations plurielles qui conjuguent créativité de la réception et réflexivité critique ? Lieu de formation de soi, de construction identitaire et culturelle, la lecture subjective sollicite des compétences qu'elle développe tout à la fois : capacité attentionnelle au texte et à soi lisant, ouverture à l'altérité, curiosité et aptitude au questionnement, exercice de la pensée critique.
\end{abstract}

Mots-clés: Enseignement de la littérature, sujet lecteur, lecture subjective.

Resumo: A leitura subjetiva designa uma forma de leitura literária que distancia abordagens exclusivamente formalistas da literatura. Ela requer a participação do sujeito-leitor e leva em conta a maneira singular na qual ele se apropria do texto e o remodela investindo em sua personalidade profunda, seus valores, seu imaginário. Esta reflexão se fez sobre a importância heurística, a riqueza, a complexidade do vivido subjetivo na experiência de leitura. Como se exprime a subjetividade do sujeito? Tecemse quais fios entre a emoção e a cognição? Enfim, como as reações sensiveis dos leitores conduzem às interpretações plurais que conjugam criatividade da recepção e reflexividade crítica? Lugar de formação de si, de construção identitária e cultural, a leitura subjetiva solicita competências que ela desenvolve ao mesmo tempo: capacidade de atenção ao texto e a si lendo,

\footnotetext{
${ }^{1}$ Ce texte est issu de la conférence faite au XVIlème GELNE nov./2018 à Recife.
} 
abertura à alteridade, curiosidade e aptidão ao questionamento, exercício do pensamento crítico.

Palavras chave: Ensino de literatura, sujeito leitor, leitura subjetiva

\section{Introduction}

\begin{abstract}
Le champ de la lecture, c'est celui de la subjectivité absolue [...]: toute lecture procède d'un sujet, et elle n'est séparée de ce sujet que par des médiations rares et ténues, l'apprentissage des lettres, quelques protocoles rhétoriques, au-delà desquels très vite, c'est le sujet qui se retrouve dans sa structure propre, individuelle.

(Roland Barthes, 1984, p. 47)

II n'existe pas de texte littéraire indépendant de la subjectivité de celui qui le lit. II est utopique de penser qu'il existerait un texte objectivable, sur lequel les différents lecteurs viendraient se projeter. [...] C'est le lecteur qui vient achever l'œuvre et refermer le monde qu'elle ouvre et il le fait chaque fois de façon différente.

(Pierre Bayard, 1998, p. 128)
\end{abstract}

La subjectivité est consubstantielle à la lecture. Tout à la fois nécessité fonctionnelle et source d'une réception créative, la subjectivité est présente dans toute lecture. Tous les chercheurs aujourd'hui en conviennent. Dès lors, pourquoi cette désignation tautologique de lecture « subjective »?

Cette insistance est volontaire. Ce qui légitime (explique) la présence de I'adjectif, c'est précisément la volonté de réhabiliter la subjectivité trop longtemps en butte aux critiques et dénigrée. II s'agit de mettre en relief une démarche qui invite à la lucidité et considère comme un fait incontournable et positif l'expression de la subjectivité des sujets lecteurs dans la lecture. Cette démarche s'inscrit en réaction à une tradition scolaire et universitaire - encore vivante - qui suspectait la subjectivité d'être à la source des erreurs, des contresens, des délires interprétatifs et la traquait, l'évinçait pour viser une objectivité du sens. Elle s'oppose ainsi à une conception instrumentale, formaliste, de l'enseignement de la littérature, inspirée du structuralisme et de la sémiotique et dans laquelle, à l'école, selon la formule saisissante de Barthes (1984, p. 40-41) : «le geste de lire disparaît sous l'acte d'apprendre ». Cette 
démarche refuse également la réification des pratiques, ce que Julien Gracq (1980, p. 171-172) désigne par «le sédiment pédagogique » qui marque l'approche des textes en privilégiant l'explication de l'œuvre sans souci aucun de la «libre imprégnation qui permet d'en jouir ».

Finalement, il importe de rétablir le sujet au cœur de la lecture : c'est lui qui donne vie au texte littéraire et lui imprime sa forme singulière. La lecture subjective dans laquelle le lecteur engage tout son être peut se définir comme un acte de métissage entre le texte de l'auteur et l'imaginaire du lecteur.

Afin de décrire ce phénomène d'appropriation du texte par le lecteur, il convient d'identifier les éléments constitutifs de la subjectivité et d'observer comment ils se manifestent durant l'acte de lecture. Mais si, dans le processus de lecture, le lecteur altère le texte, la réciproque est vraie : le lecteur sort transformé de sa rencontre avec le texte et les effets du texte sur l'intériorité du lecteur, ce que l'on peut désigner par son vécu subjectif, méritent une attention particulière. En effet, de cette expérience littéraire naît un savoir particulier dont la force tient à son origine même. Enraciné dans le corps du lecteur, dans sa mémoire vive, ce savoir reste actif dans ses choix de vie.

Selon la place accordée à la subjectivité, le pouvoir de la lecture subjective sur le sujet lecteur peut être considérable. Cette remarque conduit à poser la question des limites assignables à la subjectivité en fonction des lieux et des modalités de lecture espace privé vs espace public; lecture pour soi vs lecture scolaire, cadrée par des attentes institutionnelles et sociales.

\section{La subjectivité à l'œuvre : du texte de l'auteur au texte du lecteur \\ 1. 1 Contour de la subjectivité}

La subjectivité est ce qui appartient en propre à un sujet et s'origine en premier lieu dans ce qui est unique : son corps. C'est ce qui a fait dire à Jacques Leenhardt (1987, p. 310) : «que le corps humain dans sa totalité constitue le siège du processus de lecture » et à Michel Picard (1989, p. 133) : « le vrai lecteur a un corps. II lit avec ».

Et de fait, c'est parce que le lecteur empirique n'est pas un être désincarné qu'il réagit avec sa sensibilité aux sollicitations du texte : sensations, émotions, impressions, tout le champ des affects, est relié au corps du sujet lisant. Il en va de même de la partie 
souterraine de l'être liée à l'inconscient : pulsions et fantasmes activés par la lecture entrent également dans les composantes de la subjectivité. Quant à l'activité imageante propre à l'imagination, elle a aussi partie liée avec le corps du sujet dans la mesure où l'activité lectrice gomme les frontières traditionnellement établies entre les différentes facultés humaines :

Dans l'activité lectrice concrète, le travail imaginaire dépasse constamment les limites déterminées par les modalités du fonctionnement de l'intelligence humaine. S'il les dépasse, c'est parce que la lecture, comme activité spécifiquement humaine, met en jeu la totalité des aptitudes qui sont les nôtres. (LEENHARDT,1987, p. 310).

Donnée incontournable, l'importance du corps dans la réception des œuvres est désormais reconnue et interprétée par les chercheurs tout autant en termes d'expressivité (le corps est le siège des sensations et des émotions) que d'appropriation, d'absorption, d'incorporation du texte par le lecteur. Dans l'approche sensible de la lecture subjective, l'oralité d'un texte et la gestuelle qui l'accompagne peuvent ainsi relever de l'incorporation. C'est conscient de cet enjeu que Helder Pinheiro analyse sa pratique de la littérature de cordel et de la poésie oralisée en classe en citant Paul Zumthor :

Toute parole poétique aspire à se dire, à être entendue, à passer par ces voies corporelles qui sont celles mêmes par lesquelles s'absorbent - et j'y reviens, car c'est une analogie profonde - la nourriture, la boisson : je mange mon pain et je dis mon poème, et tu entends mon poème de la même façon que tu entends les bruits de la nature (1990, p. 60).

La subjectivité du sujet est aussi façonnée par son environnement social. Le lecteur vient au texte avec des représentations, un univers de croyances construit au fil de son histoire personnelle. Les références issues de sa connaissance du monde et de la littérature orientent sa réception du texte. Toutefois, il convient de rappeler, à la suite de Jauss, que s'il existe bien un filtre personnel qui sélectionne et transforme les éléments du texte, la lecture pragmatique ne renvoie pas au réel mais à une reconstitution du réel par des stéréotypes. Dès lors, l'adoption de scénarios conventionnels, stéréotypés, qui se substituent à la réalité et lui font écran, intègre le lecteur à une communauté, gommant non sa subjectivité mais sa singularité. 
L'interprétation d'un texte met en jeu les facultés intellectuelles du lecteur mais aussi son axiologie, son idéologie, sa culture qui donnent une couleur spécifique à sa pensée. La rationalité, les inférences, toutes les opérations cognitives nécessaires aux démarches heuristiques et herméneutiques n'éradiquent donc pas pour autant la subjectivité. L'interprétation est toujours un choix, l'expression d'une préférence personnelle parmi les significations possibles du texte. Toute pensée « vivante » porte l'empreinte de son auteur ; toute énonciation fait entendre une inflexion singulière. Ainsi le lecteur empirique aborde-t-il le texte avec sa sensibilité et son intelligence ; sa réception met en jeu son vécu, sa culture, son inconscient.

\subsection{La subjectivité à l'œuvre dans l'appropriation du texte}

Durant la lecture, le lecteur investit le texte de l'auteur et le transforme à son image, il le singularise. Par son activité créatrice faite d'ajouts, d'effacements, de déplacements, le lecteur, écrit W. Iser (1985, p. 198), prend « une part égale au jeu de I'imagination ». À partir du texte de l'auteur, il crée son «texte de lecteur », notion théorisée dès 1998 par Pierre Bayard dans Qui a peur de Roger Acroyd? : "Chaque lecteur constituant son propre réseau d'indices - ce n'est pas le même texte qui est lu » (p. 90).

Jean Bellemin-Noël (2001) dans une perspective psychanalytique, souligne l'importance de cette appropriation en précisant que le texte ainsi créé réfracte les intérêts conscients ou inconscients du lecteur. Finalement, si l'on peut parler de texte du lecteur, ce n'est pas seulement parce que le texte est remodelé par le lecteur mais bien plus encore parce que le lecteur est présent dans le texte qu'il produit. Et il évoque

Ce trajet de lecture qui seul, peut-être mériterait d'être appelé texte, et qui est tissé par la combinaison fluctuante de la chaîne de [sa] vie avec la trame des énoncés une fois pour toute combinés par l'auteur. (NOEL, 2001, p. 21)

L'imagination du lecteur joue un rôle essentiel dans cette métamorphose qui s'apparente à un métissage. Celle-ci s'alimente à diverses sources. Elle puise dans un répertoire hétérogène d'images qui proviennent de l'expérience du monde du lecteur : à la fois de son histoire personnelle (ses souvenirs) et de l'imaginaire collectif (motifs et 
stéréotypes) de la société dans laquelle il vit. L'imagination sollicite également des images perçues ou formées lors d'expériences esthétiques antérieures, notamment celles qui sont liées à des souvenirs littéraires, ceux-là mêmes qui jailliront appelés par le phénomène d'interlecture. Cette notion, créée par J. Bellemin Noël (2001, p. 12-13), désigne le rapprochement de deux œuvres, librement effectué par le lecteur, hors de tout indice d'intertextualité. Dans son activité de représentation, le lecteur fait donc feu de tout bois, mais les images mentales qui se présentent à la lecture sont, selon la terminologie d'Husserl reprise par Iser, « des synthèses passives » (ISER, 1985, p. 253260), ce qui signifie qu'elles naissent en-deçà du seuil de la conscience, qu'elles sont indissociables du sujet qui les a fait naître et tirent leur force de l'écho qu'elles suscitent dans les profondeurs de l'être. Pourtant, au fil de la lecture, nombre d'images s'effacent; le texte du lecteur, quand il n'est pas saisi par l'écriture est un objet immatériel, fragile, éphémère qui a tendance à s'effacer ou à se cristalliser avec le temps.

Évoquant l'imaginaire du lecteur, j'ai mis l'accent sur cette floraison d'images, qui est en quelque sorte son matériau privilégié, mais l'imagination est également ce qui dirige la construction du sens et l'interprétation. Dans l'incipit de L'Espèce fabulatrice, Nancy Huston (2008, p. 11) rappelle que «c'est notre imagination qui confère au réel un Sens qu'il ne possède pas par lui-même. " Au-delà de l'activité de représentation, l'imagination du lecteur réécrit le texte et procède ainsi à sa «reproduction, re-fictionnalisation ». Gérard Langlade (2006) s'est intéressé à "l'activité fictionnalisante du lecteur " qu'il décrit à l'aide des quatre opérations suivantes : la « concrétisation imageante » pour donner corps à l'univers diégétique; le souci de la cohérence mimétique, garante de la lisibilité du texte ; la configuration axiologique par laquelle le lecteur investit l'univers fictionnel de ses propres valeurs et la mise en œuvre de scénarios fantasmatiques.

Ainsi dans le processus de lecture, le sujet lecteur altère le texte en lui imprimant sa singularité. Cet acte d'appropriation qui transforme le texte de l'auteur en texte du lecteur échappe à sa conscience. La réciproque est moins vraie : si l'altération du lecteur par le texte est un processus très souvent inconscient, il est des expériences de lecture inoubliables, qui laissent des traces profondes dans la vie des lecteurs. 


\section{La richesse du vécu subjectif}

Décrire ce qui se passe dans l'intériorité du lecteur relève de la gageure. Les chercheurs peuvent prendre appui sur des témoignages de lecteurs, mais ceux-ci, comment peuvent-ils rendre compte de ce qui se dérobe le plus souvent à l'analyse ? Le sujet est le plus souvent opaque à lui-même, au moins en ce qui concerne certains besoins existentiels présents en lui et qu'il ne soupçonne pas. « Il existe en tout être un 'infracassable noyau nuit' ", écrit S. de Beauvoir (1960, p. 150), reprenant l'image d'André Breton.

L'expérience du vécu fictif qui plonge le lecteur dans l'univers du texte est souvent gage d'enrichissement en termes de savoir sur l'homme et sur le monde. La lecture de romans impose un dépaysement qui transporte le lecteur dans des mondes fictifs qui l'ouvrent à des valeurs ou des modes de pensée nouveaux. Ceci est vrai de toute lecture qui confronte nécessairement à l'altérité, mais cette altérité varie en intensité selon l'origine du texte lu (classiques et littérature du passé ; littérature étrangère; littérature d'avant-garde etc.). Selon Iser, (1985, p. 284) «l'assimilation d'éléments étrangers ne se fait que si la conscience elle-même accepte de prendre une nouvelle forme. " La restructuration du savoir qui s'opère alors peut s'interpréter comme un enrichissement de la vision du monde.

Plus essentielle encore, est l'expérience de l'identification par laquelle le lecteur élargit son être, explore des moi possibles, découvre en lui des zones d'ombre. Loin d'être synonyme de passivité et de régression, et même d'aliénation comme on l'a longtemps pensé, ainsi que le rappelle Jauss (1978, p. 132) : "l'identification est un phénomène complexe, une expérience troublante. Elle implique du sujet la capacité à sortir de soi-même pour découvrir l'autre qui est en lui ». Ainsi que le décrit Ricœur (1986) le lecteur se « désapproprie de [lui]-même pour se laisser être la chose du texte » (1986, p. 54), car finalement, " se comprendre, c'est se comprendre devant le texte et recevoir de lui les conditions d'un soi autre que le moi qui vient à la lecture ". (1986, p. 31)

Chaque lecture transforme le lecteur; elle accroît son expérience humaine, modifie son identité ; celle-ci est une construction infinie. L'identification est le siège d'un processus actif au cours duquel le lecteur réagit par « une réponse productive à une 
différence vécue » entre «ce texte en moi présent et mon expérience repoussée dans le passé » (ISER, 1985, p. 241).

Cependant, bien qu'il soit acteur de son propre changement, le plus souvent, le lecteur n'a pas conscience de ce qui advient dans le vécu de la lecture, au-delà des réactions de plaisir ou de déplaisir. Nombre de livres lus rejoignent ainsi la bibliothèque intérieure du sujet sans lui laisser de traces vives en mémoire. II faut que le bouleversement soit intense, voire violent et provoque ce que l'on nomme un évènement de lecture, pour que le lecteur en perçoive les effets.

L'un des témoignages les plus forts est sans doute celui de G.-A. Goldschmidt (2004, p. 52), relatant, dans son autobiographie de lecteur Le Poing dans la bouche, la commotion produite en lui par la lecture de Le Procès de Kafka, l'intuition fulgurante d'un moment décisif : « La première phrase fut un véritable coup de boutoir en pleine poitrine, elle me coupa le souffle, d'emblée, je sus que j'avais trouvé enfin mon livre ». En quête de sa propre vérité, Goldschmidt (2004) cherche à assumer son identité déchirée - sa condition de Juif, ses penchants masochistes. Le roman de Kafka a finalement l'effet cathartique d'une lecture libératrice, apaisante, permettant un accomplissement :

Une vie se construit largement sur des lectures fondatrices, sur des lectures de rupture qui inversent le cours de la normalité, qui ramènent d'en dessous de soi-même, ce qu'on n'osait pas même formuler, à quoi il était interdit de penser. (GOLDSCHMIDT, 2004, p. 101)

D'autres témoignages saisissants confirment l'extraordinaire pouvoir de certaines identifications empathiques. Ainsi, dans Un ange cornu avec des ailes de tôle, I'écrivain québécois Michel Tremblay raconte :

Quand je terminai Agamemnon [...], j'eus l'impression d'être devenu quelqu'un d'autre, d'avoir grandi, évolué en quelques heures, d'avoir entrevu des possibilités qui me concernaient personnellement et qui transformeraient ma vie d'une façon définitive, j'ignorais encore quoi, mais ça m'était entré dans le corps, dans le cœur pour le reste de mes jours. (TREMBLAY, 1996, p. 211)

Simone de Beauvoir, dans Les Mémoires d'une jeune fille rangée $(1958,124-125)$ raconte comment son identification passionnée à Joe, personnage de Little Women de Louisa Alcott, a ouvert la voie à une émancipation déterminante pour son avenir 
d'intellectuelle et d'écrivain. André Gide a connu une expérience semblable, et dans une conférence sur la lecture, il évoque les livres ou les quelques phrases d'un livre qui s'incorporent à nous et dont la puissance « vient de ceci qu'elle n'a fait que me révéler quelque partie de moi-même inconnue à moi-même; elle n'a été pour moi qu'une explication de moi-même ». [...] Et il ajoute, évoquant le conte de Perrault La Belle au bois dormant : «Combien de sommeillantes princesses nous portons en nous, ignorées, attendant qu'un mot les réveille. $»^{2}$

Dans ces expériences fondatrices, le sujet accède à lui-même, découvrant dans le texte un secret enfoui en lui. "Cette lecture savait quelque chose de moi que personne ne devait savoir ; elle savait de l'inavouable... ", écrit Goldschmidt (2004, p. 67). Finalement, le lecteur est lu par le texte et, précisément, si l'on se réfère à la théorie de Michel Picard dans La lecture comme jeu (1986), c'est le lu - instance où s'expriment les pulsions inconscientes - qui est mis en lumière dans ces récits autobiographiques.

\section{Un savoir spécifique, intuitif et expérientiel}

Dans sa « Leçon inaugurale au Collège de France », Antoine Compagnon (2018) présente la littérature comme le lieu d'un savoir unique, issu d'une expérience unique : [...] elle renferme un savoir irremplaçable, circonstancié et non résumable sur la nature humaine, un savoir des singularités. La littérature exprimant l'exception, procure une connaissance différente de la connaissance savante mais mieux capable d'éclairer les comportements et les motivations humaines. (COMPAGNON, 2018, p.61).

Ce savoir issu de l'expérience de lecture s'oppose à la pensée conceptuelle, abstraite et généralisante. Dans sa Petite apologie de l'expérience esthétique, Jauss (1978), s'intéresse la spécificité de ce savoir qu'il décrit, à partir des concepts clés de la tradition esthétique, comme «un savoir également distinct de la connaissance scientifique, conceptuelle et de la praxis artisanale purement reproductrice, limitée par sa finalité (1978, p. 131) mais aussi un savoir qui rend à l'intuition « ses droits contre le privilège accordé traditionnellement à la connaissance conceptuelle » (1978, p. 131). Le

\footnotetext{
${ }^{2}$ Conférence sur la lecture, citée par Pierre Lepape, in : Le Monde du 15/10/99 http://enfants-du-net.org/ takalir/projo/index. Php 3 ? contenu=petit
} 
faible degré d'abstraction de ce savoir n'enlève rien à sa qualité et Jauss se plait à rappeler que, dans son Faust, Goethe affirme la supériorité de la fonction cognitive de la jouissance esthétique sur l'abstraction du savoir conceptuel.

Dans l'expérience esthétique, les modes d'activité de la conscience mêlent à des degrés divers sensibilité et cognition. Cette intrication profonde entre émotion est cognition est une donnée d'importance sur laquelle insiste Vincent Jouve dans L'Effetpersonnage dans le roman : il insiste ainsi sur la dimension cognitive de l'identification, souvent méconnue des lecteurs eux-mêmes, sensibles surtout à l'émotion ressentie. « Le primat de l'émotion - écrit-il - entraîne ainsi une expérience complexe où sensation et réflexion se trouvent inextricablement mêlées " (JOUVE, 1992, p. 198).

Le complexe de réactions émotives et cognitives qui surgit à la lecture est un moment précieux, fragile, souvent éphémère qui se laisse difficilement appréhender avec les mots, toujours réducteurs. Savoir tissé d'affects, affects porteurs de savoir, comment démêler l'écheveau ? Enracinement réciproque des deux composantes l'une dans l'autre - tout comme aujourd'hui on admet la porosité de la compréhension et de I'interprétation - simultanéité de leur émergence, c'est à partir de ce chaos que le sujet lecteur doit construire sa pensée.

La distanciation propre à la réflexivité intervient a posteriori lorsque sujet lecteur se détache de son livre. Dans Le Pain des rêves, Louis Guilloux adulte revient sur ses lectures d'enfance et la conception qu'il était forgée de la littérature :

La croyance naïve s'était formée en moi que les poètes étaient des personnages aussi légendaires que ceux de l'histoire sainte, qu'ils avaient existé au temps des fables et que, depuis, on n'en avait jamais plus revu, qu'on n'en reverrait plus jamais. Victor Hugo, dont je savais par cœur Après la bataille, vivait d'après moi dans une sorte d'Olympe, en tout cas sans rapport avec l'humanité de la rue du Tonneau. Pour tout dire, il n'était qu'un beau et grand mensonge. (GUILLOUX, 1942, p. 98-99)

De la distance entre le monde fabuleux décrit par la littérature et la réalité vécue connue de l'enfant, naît une réflexion sur les stéréotypes littéraires et leur contraste avec son expérience du monde. Dans cette évocation du travail psychique né de la lecture, on perçoit comment le sujet construit sa pensée, développe son esprit critique et prend conscience de sa place dans le monde. Apparaît ainsi dès l'enfance une conscience de classe qui ne le quittera pas et motivera son travail d'écrivain. 
La réflexivité à l'œuvre évoquée par Louis Guilloux - et sans doute reconstruite par son travail d'écriture - est une expérience partagée par nombre de lecteurs qui n'en rendent pas compte spontanément car elle touche à l'intime.

À l'écoute de lecteurs, I'anthropologue Michèle Petit revient sur ce phénomène :

Ce qui est saisissant, à écouter ces lecteurs, ces lectrices, c'est l'évocation du travail psychique, du travail de rêverie, de pensée qui a accompagné ou suivi la lecture. [...] C'est l'élaboration d'une position de sujet qui est en question. D'un sujet qui construit son histoire en prenant appui sur des fragments de récits, des images, des phrases écrites par d'autres, et qui en tire force, quelquefois, pour aller ailleurs que là où tout semblait le destiner. (PETIT, 2002, p. 4)

On le voit, dans tous ces témoignages, la réflexivité donne sens au ressenti de la lecture et éclaire ses incidences identitaires sur le sujet. Mais les expériences évoquées sont des lectures privées dans lesquelles le lecteur peut utiliser (Eco, 1996) le texte, sans contrainte, pour se penser et penser le monde.

Dans un cadre institutionnel - l'école, l'université - des limites sont posées à la subjectivité. Vincent Jouve (2004) les évoque en distinguant subjectivité nécessaire requise par le texte et subjectivité accidentelle, libre création du lecteur. On ne saurait donc, dans une perspective de formation du lecteur éluder cette question de la place dévolue à la subjectivité dans la lecture.

\section{Limiter la subjectivité ? Espaces sociaux et formation des lecteurs}

La richesse des expériences de lecture tient souvent à leur survenue dans la sphère intime. Ce qui frappe également, c'est leur caractère imprévisible. Alberto Manguel dans son Journal d'un lecteur met en lumière le rôle des coïncidences - au double sens de hasard et d'adhésion - dans la rencontre avec les œuvres, coïncidence qui se traduit par l'existence, à un moment donné, d'une conjonction entre une œuvre et la disposition d'un sujet. Celui-ci s'approprie librement le texte en en mettant certains détails en rapport avec la vie :

Pour qu'un livre nous touche, il faut sans doute qu'il établisse entre notre expérience et celle de la fiction - entre deux imaginations, la nôtre et celle qui se déploie sur la page - un lien fait de coïncidence. (MANGUEL, 2004, p. 32) 
Il s'agit là d'un usage tout personnel de la lecture, semblable en cela aux manières singulières de lire des lecteurs décrits par I. Calvino (1981) dans Si par une nuit d'hiver, un voyageur.

L'école, en revanche uniformise (ou tend à uniformiser) les manières de lire, limitant fortement la liberté des sujets lecteurs. L'objectif de la formation des lecteurs étant l'élaboration d'une posture distanciée et critique censée permettre un accord des esprits sur une signification, la place laissée à la subjectivité est limitée par les « droits du texte ", ou, si l'on préfère, à la seule subjectivité nécessaire. Pendant longtemps, et parfois encore aujourd'hui, la réponse à cette attente institutionnelle s'est traduite à défaut d'un « cadrage » de la subjectivité par son éradication pure et simple.

Aujourd'hui, la situation est différente. Consciente de la désaffection des élèves pour la littérature, I'Institution recommande la prise en compte de la réception des élèves et la lecture subjective commence à prendre place dans les classes. ${ }^{3}$

Paradoxalement l'un des principaux problèmes rencontrés par les enseignants de collège et de lycée n'est pas l'existence d'une subjectivité débridée mais au contraire le mutisme des élèves qui n'osent pas s'exprimer. Diverses raisons expliquent ce comportement : la pudeur liée au dévoilement de soi dans un cadre collectif où chacun s'évalue ; le manque de mots pour dire ce qui est compris et ressenti ; ou, inversement, le vide que certains élèves trouvent en eux. II faut donc les amener à se considérer comme des sujets lecteurs libres de s'exprimer et disposant d'une culture littéraire dont ils peuvent prendre conscience en réalisant leur propre autobiographie de lecteur. Cela suppose aussi le respect de leurs lectures privées.

La pratique de la lecture subjective en classe suppose donc de créer les conditions d'émergence et de construction de la subjectivité qu'on ne saurait assimiler illusoirement aux seules réactions spontanées et intempestives des jeunes lecteurs. Des dispositifs ont été imaginés ( $N$. Brillant-Rannou, 2011) pour permettre aux élèves de capter et d'identifier leur ressenti, en leur proposant notamment des répertoires de mots qui les questionnent et les aident à aller plus profondément en eux-mêmes. Les

\footnotetext{
3 Voir XYPAS, R. Por uma didática da implicação do leitor na leitura do texto literário. In: XYPAS, Rosiane. A leitura subjetiva no ensino da Literatura: Apropriação do texto literário pelo sujeito leitor. Olinda: Nova Presença, pp. 73-98, 2018.
} 
élèves s'étonnent souvent de se découvrir dans cette activité de lecture et, au-delà du savoir-faire, cette expérience construit leur motivation.

Les recherches récentes en didactique de la littérature (B. Shawky-Milcent, 2016 ; S. Lemarchand, 2017) soulignent l'importance du questionnement de soi dans I'élaboration du sens. Quand I'attention du lecteur ne se limite pas au jeu des formes textuelles et s'intéresse au « soi lisant », autrement dit, quand le lecteur est à l'écoute de lui-même, les hypothèses de sens proposées sont plus fécondes et cette expérience renforce le goût de lire. Ces recherches montrent aussi l'efficacité de consignes simples qui interrogent l'imaginaire du lecteur, sollicitent sa créativité, son inventivité notamment dans des activités d'écriture ou par la mise en écho avec d'autres œuvres, littéraires, plastiques ou musicales. Elles s'intéressent à toutes les modalités appropriatives des œuvres et à leur signification et n'hésitent à importer en classe des formes de lectures privées. Ainsi, la lecture «voyageuse, dansante », lecture de braconnage, évoquée Michel de Certeau (1980) est-elle interrogée et envisagée comme une recréation, une forme d'appropriation singulière du texte par le lecteur. De même les pratiques de « bricolage » effectuées sur les textes sont appréhendées positivement dans ce qu'elles signifient comme formes d'appropriation originales. Des exemples précis de dispositifs innovants en lien avec les pratiques sociales des lecteurs, comme «L'écriture dans les marges » qui permet un dialogue entre deux lecteurs, ou encore, «Écrire à quatre mains » (celles de l'auteur et celles du lecteur) qui repose sur le geste anthologique et compose une nouvelle œuvre à partir d'extraits sélectionnés par le lecteur ont été imaginés par Nathalie Rannou (2016). Plus en phase encore avec I'univers culturel des jeunes, des activités de «booktube » ${ }^{4}$ et de «litteratube » dédiée au partage de lectures par la création de vidéos peut se révéler particulièrement féconde. Ainsi en est-il, par exemple, de la réalisation de Dorian Moulard, étudiant de master recherche en littérature ${ }^{5}$, réalisation à la fois déroutante par le choix d'images abstraites et impressionnante par le commentaire associé : « Rien n'est plus réel que ce qui se joue dans mon esprit ».

\footnotetext{
4BRILLANT- RANNOU N. voir : https://litmedmod.ca/sites/default/files/pdf/r2-Imm_vol8_rannou.pdf 5 Étudiant de l'Université de Rennes 2, en 2018, https://www.YouTube.com/watch?v=UpTMp7SkdXA\&feature=youtu.be
} 
Toutes ces pratiques invitent à plus de souplesse. Finalement plutôt de que limiter autoritairement la subjectivité des lecteurs, il est plus fructueux de l'accueillir, y compris dans ses débordements et de solliciter l'esprit critique des élèves en les invitant à débattre des propositions sémantiques, à partager leurs lectures. L'intersubjectivité présente dans la communauté interprétative qu'est la classe est à même de réguler la subjectivité des participants.

Finalement, ce qui importe, c'est de créer les conditions pour que les élèves se sentent concernés par la littérature et puissent vivre en classe (ou grâce à la classe) une véritable expérience intérieure.

\section{Conclusion}

La lecture subjective suppose la liberté du lecteur, une liberté assumée et réfléchie. Elle déborde souvent les limites que lui impose l'école et qui finalement la dénature. De fait, le lecteur investit le texte de sa propre vie mais, ainsi que l'écrit G. Langlade (2004, p. 85) : « les réactions subjectives loin de faire tomber les œuvres « hors de la littérature » seraient des catalyseurs de lecture qui alimenteraient le trajet interprétatif jusque dans sa dimension réflexive ». En effet, la lecture subjective impulse la réflexion; les réactions personnelles sont sources d'un questionnement qui dépasse les effets produits par le texte pour s'intéresser à ses enjeux, aux significations qui motivent son existence.

Lieu d'une expérience esthétique la lecture subjective est aussi un lieu de formation de soi. Elle sollicite des compétences qu'elle développe tout à la fois: capacité attentionnelle au texte et à soi-même, ouverture à l'altérité, aptitude au questionnement, exercice de la pensée critique.

Mais pour que les lectures subjectives puissent advenir en classe, il importe de préserver ou de ménager pour les élèves des moments d'intimité avec le texte, espaces de liberté, de création.

La disposition inventive est affaire de liberté, de décrochement, de détour, de régression vers des liaisons oniriques, de diminution des tensions. [...] Dans le contact avec les œuvres artistiques, cet autre registre est touché: "une pensée en mouvement, inventive et déliée ", toute en "connexions inattendues", qui transgresse les interdits, pérégrine. La condensation, le déplacement, ces modes de fonctionnement propres à l'inconscient, aux « processus primaires, 
peuvent alors trouver passage jusqu'à la pensée rationnelle. » (PETIT, 2002, p. 43-44, citant PONTALIS, 2001, p. 235-243)

Le travail collectif de réflexion et d'analyse sur les lectures subjectives accueillies en classe, s'il est conçu comme un partage, débouche non sur une lecture consensuelle érigée en modèle, mais sur un ensemble de propositions singulières enrichies par la communauté, et témoin de la polysémie du texte.

Finalement, la lecture subjective, telle que nous l'avons décrite, répond aux aspirations humanistes et démocratiques d'Antonio Candido, en ouvrant à tous un accès à la littérature.

\section{Bibliographie}

BARTHES, R. Sur la lecture. In : Le Bruissement de la langue. Essais critiques IV, Points Seuil, 1975/1984.

BEAUVOIR, S. de. Les Mémoires d'une jeune fille rangée. Paris : Gallimard, Coll. Folio, 1958.

La Force de l'âge. Paris : Gallimard- Folio, 1960.

BAYARD, P. Qui a tué Roger Acroyd ? Paris : Minuit, 1998.

BELLEMIN-NOËL, J. Plaisirs de vampire. Paris : PUF, 2001.

BRILLANT-RANNOU, N. Questions sur le texte du lecteur de poésie. In: MAZAURIC, C.; FOURTANIER, M-J.; LANGLADE, G. (dir). Textes de lecteurs en formation. Berne : Peter Lang, coll. ThéoCrit, vol. 3, 2011.

BRILLANT-RANNOU, N. Le recueil à quatre mains et la lecture dialoguée dans les marges : conception et expérimentation de deux dispositifs de lecture subjective de poésie. In: BRILLANT-RANNOU, N.; BOUTEVIN, C.; BRUNEL, M. (DIR.) Être et devenir lecteur(s) de poèmes : de la poésie patrimoniale au numérique, Diptyque $n^{\circ}$ 32, Namur, Presses Universitaire de Namur, p. 99-126, 2016.

https://litmedmod.ca/sites/default/files/pdf/r2-Imm_vol8 rannou.pdf

CALVINO, I. Si par une nuit d'hiver, un voyageur... Paris: Gallimard, coll. Points Roman, n 81, 1981.

CAMBRON, M. ; LANGLADE, G. (dir.) L'Evénement de lecture, Nota Bene : Montréal, 2015 . 
CERTEAU, M. de. Lire : un braconnage. In : L'Invention du quotidien. 1) Arts de faire, Paris : $10 / 18,1980$.

COMPAGNON, A. La littérature pour quoi faire ? - Leçon inaugurale du 30 novembre 2006, au Collège de France, ( $\left.n^{\circ} 188\right)$, Fayard/Pluriel, 2018.

ECO, U. Six promenades dans les bois du roman et d'ailleurs. Paris, GrassetFasquelle, 1994/ 1996.

GIDE, A. Conférence sur la lecture- citée par Pierre Lepape. In: Le Monde du 15/10/99http://enfants-du-net.org/takalir/projo/index. Php 3 ? contenu=petit

GOLDSCHMIDT, G.-A. Le poing dans la bouche. Verdier, 2004.

GRACQ, J. En lisant, en écrivant. Paris : Corti, 1980.

GUILLOUX, L. Le pain des rêves. Gallimard, coll. Folio, 1942.

HUSTON, N. L'Espèce fabulatrice. Actes Sud, 2008.

ISER, W. L'Acte de lecture - Théorie de l'effet esthétique. Mardaga, Coll. «philosophie et langage », 1976/1985.

JAUSS, H.-R. Petite Apologie de l'expérience esthétique. In : Pour une esthétique de la réception. Paris : Gallimard, coll. Tel., 1978.

JOUVE, V. L'Effet-personnage dans le roman. Coll. Ecriture, Paris : P.U.F. 1992.

LANGLADE, G. L'activité fictionnalisante du lecteur. In: M. BRAUD, B. LAVILLE, B. LOUICHON (ed.) Les Enseignements de la fiction. Bordeaux : Modernités $n^{\circ} 23$, PUB, 2006.

Le sujet lecteur auteur de la singularité de l'œuvre. In : Rouxel A. et Langlade G. Le Sujet lecteur. Lecture subjective et enseignement de la littérature. Rennes: PUR, p. 81-91, 2004 .

LEENHARDT, J. Les instances de la compétence dans l'activité lectrice. In - Michel PICARD (éd.) La Lecture littéraire. Paris : Clancier-Guénaud, p. 302-311, 1975/1984.

LEMARCHAND, S. Devenir lecteur. L'expérience de l'élève de lycée professionnel. Rennes: PUR, 2017.

MANGUEL, A. Journal d'un lecteur (A Reading Diary), Actes Sud, collection Babel, 2004 . 
MOULARD,

D.

https://www.YouTube.com/watch?v,=UpTMp7SkdXA\&feature=youtu.be

PETIT, M. Éloge de la lecture. La construction de soi. Paris : Belin, 2002.

L'art de lire ou comment résister à l'adversité. Paris : Belin, 2008.

PICARD, M. La Lecture comme jeu. Paris : Minuit, 1986.

. Lire le temps. Paris : Minuit, 1989.

PINHEIRO, H. Poesia na sala de aula, Campinas: Parábola, 2018.

RICOEUR, P. Du texte à l'action. Essais d'herméneutique. coll. Esprit, Paris : Seuil, 1986.

ROUXEL, A. ; LANGLADE, G. Le Sujet lecteur. Lecture subjective et enseignement de la littérature. Rennes: $P U R, 2004$.

ROUXEL, A. Lecture et retour sur soi : I'autobiographie de lecteur au lycée. In - Enjeux 61, décembre, p. 47-68, 2004.

Ma vérité dans cet éclat du texte... Lectures fondatrices : révélation, a/encrage et réancrage. In: Micheline CAMBRON, Gérard LANGLADE (dir.) Le Divers dans l'évènement de lecture. Montréal : Nota Bene, p. 129- 145, 2015.

Mobilité, évanescence du texte du lecteur, In : MAZAURIC, C. ; FOURTANIER, M-J. ; LANGLADE, G. (dir.) Le Texte du lecteur. Bruxelles, Peter Lang, coll. Théocrit, p. $115-128,2010$.

ROUXEL, A., LANGLADE, G.; LUZIA DE REZENDE, N. (org.) Leitura subjetiva e ensino de literatura, Alameda, 2013.

ROUXEL, A. Oser lire à partir de soi. Enjeux épistémologiques, éthiques et didactiques de la lecture subjective :

http://revista.abralic.org.br/index.php/revista/issue/view/3

PDF, p. $10-25$.

SHAWKY-MILCENT, B. La lecture, ça ne sert à rien ! Usages de la littérature au lycée et partout ailleurs. Paris : Presses Universitaires de France, 2016.

TREMBLAY, M. Un ange cornu avec des ailes de tôle. Récits, Montréal/Arles, Leméac/Actes sud, coll. « Babel », 1996.

XYPAS, R. A leitura subjetiva no ensino de literatura: apropriação do texto literário pelo sujeito leitor, Olinda: Nova Presença, 2018. 
ZUMTHOR, P. Présence de la voix. Cinq entretiens avec André Beaudet à Radio Canada (1984) Ecriture et nomadisme. Entretiens et essais. Montréal: I'Hexagone, 1990.

Escritura e nomadismo. (Trad) Jerusa P. Ferreira e Sonia Queiroz. São Paulo: Ateliê Editorial, 2005.

\footnotetext{
'Annie Rouxel est professeur émérite de l'Université de Bordeaux (France). Elle a dirigé plusieurs travaux de recherches dont des Masters, des doctorats et accompagné des HDR. Elle est rattachée au Centre d'études des langues et littératures anciennes et modernes (CELLAM) à Rennes 2, EA326. Son axe de recherche est celui de la didactique de littérature.

E.mail : ani.rouxel@orange.fr
} 